\title{
PML Nuclear Body Identification and Ultrastructure in Rodent Tissues and Cultured Cells by Post-Embedding Immunogold Labeling.
}

Stephen Hearn*, Edith Heard ${ }^{+}$, Emmanuelle Querido, Scott Lowe and David L. Spector. Cold Spring Harbor Laboratory, 1 Bungtown Road, Cold Spring Harbor, New York 11724, USA, ${ }^{+}$Institut Curie Section de Recherche, 26 rue d'Ulm75005 Paris FRANCE, *Cell Biology, The Hospital for Sick Children, 555 University Avenue, Toronto, Ontario Canada M5G 1X8.

Interphase mammalian cell nuclei contain discrete sub-compartments called promyelocytic (PML) nuclear bodies, which are associated with cellular processes such as tumorigenesis, proliferation, differentiation, apoptosis, senescence, viral infection and neurodegenerative disease. PML bodes are defined as 200-500nm bodies present as 10-30 spherical dots in cultured cells labeled for promyelocytic leukemia protein by fluorescence microscopy. PML nuclear bodies are believed to be a minor sub-class of a larger set of diverse Nuclear Bodies that were identified by morphological means alone in classic electron microscopic studies in the 1960's. In order to conveniently identify PML in optimally preserved tissue and cultured cells, we applied a postembedding immunogold labeling approach using monoclonal antibodies to PML protein.

Hybridoma clone supernatants were screened by immunofluorescence on cultured mouse embryonic fibroblasts (MEFs) and by immuno-blotting. Positive clones were selectively tested on ultrathin sections of mouse and rat liver, and cultured cells (BHK, Mc Coy cells, and mouse embryonic stem cells (ES); antibody binding was detected using goat antimouse IgG conjugated to 5 or $10 \mathrm{~nm}$ colloidal gold (Electron Microscopy Sciences.). Samples of mouse cells and tissue were fixed in either $4 \%$ formaldehyde or $1.6 \%$ glutaraldehyde and then embedded in Lowicryl K4M and UV cured at $-35^{\circ} \mathrm{C}$. Two IgG supernatants produced positive and specific labeling of characteristic Nuclear Bodies in nuclei of cultured cells and as well as nuclei in the liver tissues. A great variety of nuclear body types were represented in the positively labeled structures and no labeling was present over other nuclear compartments such as Cajal bodies, or interchromatin granule clusters.

Fluorescence techniques using wide field, confocal or live-cell microscopy have shown that PML bodies can associate with active chromatin regions, RNA transcripts, Cajal bodies, nucleoli, and viruses. Using immunogold labeling for PML protein should be of value in understanding the roles of Nuclear Bodies in gene regulation and RNA transcription.

References:

(1) M Bouteille et al., J. Ultrastruct. Res. 19 (1967) 474.

(2) P Solomoni and PP Pandolfi. Cell 108 (2002) 165. 


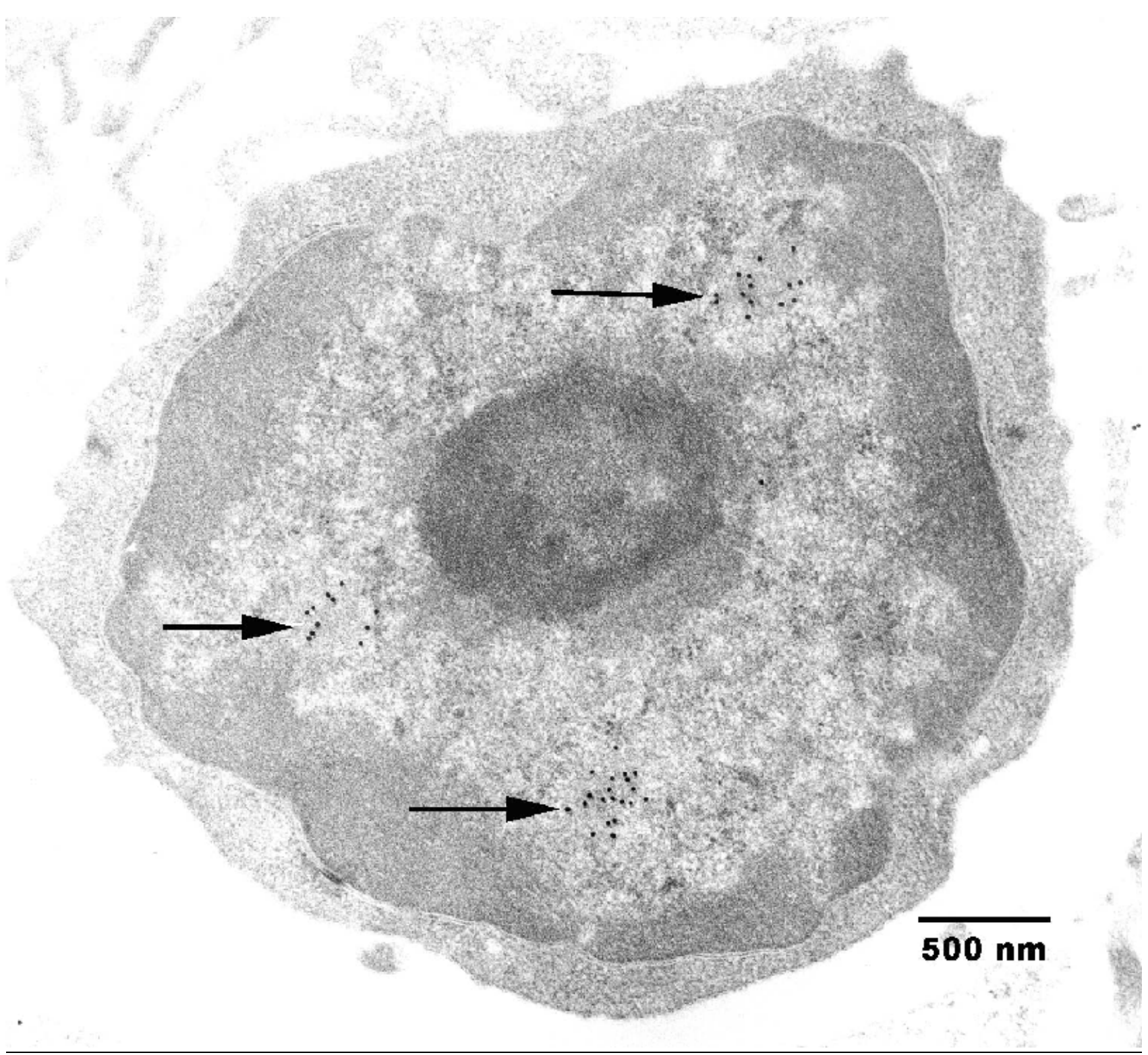

Figure 1

Immunogold labeling for PML protein over Nuclear Bodies (arrows) in lymphocyte in mouse liver 